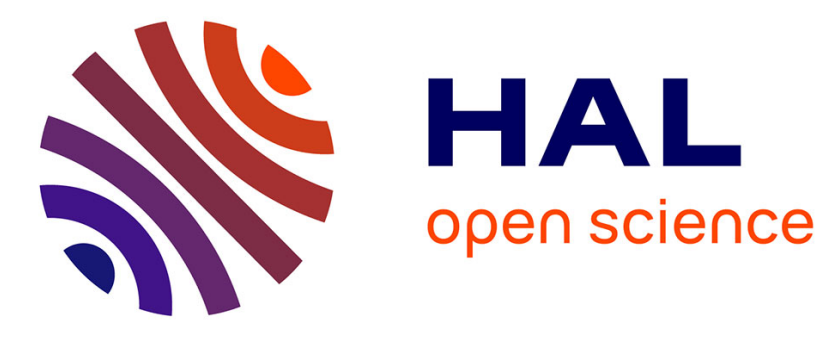

\title{
Mesure des flux infrarouges dans la troposphère
}

M. Roulleau

\section{- To cite this version:}

M. Roulleau. Mesure des flux infrarouges dans la troposphère. Revue de Physique Appliquée, 1979, 14 (1), pp.159-163. 10.1051/rphysap:01979001401015900 . jpa-00244572

\section{HAL Id: jpa-00244572 https://hal.science/jpa-00244572}

Submitted on 1 Jan 1979

HAL is a multi-disciplinary open access archive for the deposit and dissemination of scientific research documents, whether they are published or not. The documents may come from teaching and research institutions in France or abroad, or from public or private research centers.
L'archive ouverte pluridisciplinaire HAL, est destinée au dépôt et à la diffusion de documents scientifiques de niveau recherche, publiés ou non, émanant des établissements d'enseignement et de recherche français ou étrangers, des laboratoires publics ou privés. 


\title{
Mesure des flux infrarouges dans la troposphère
}

\author{
M. Roulleau \\ Laboratoire de Météorologie Dynamique, Ecole Polytechnique, \\ 91128 Palaiseau Cedex, France
}

(Reçu le 30 juin 1978, accepté le 10 octobre 1978)

\begin{abstract}
Résumé. - On expose le projet concernant l'étude de l'influence du profil de vapeur d'eau sur les flux infrarouges dans la troposphère. Le but est d'établir une relation paramétrique entre ces flux et les paramètres aérologiques classiques. Le dispositif expérimental utilisé pour les sondages radiométriques de la troposphère est décrit en détail.

Abstract. - This paper describes a project concerning the influence of water vapor profile on the tropospheric infrared fluxes. The purpose is to establish a parametric law between these fluxes and the classical meteorological parameters. The technique used for the radiometric soundings of the troposphere is described in details.
\end{abstract}

1. Introduction. - La représentation de la divergence du flux radiatif dû au rayonnement de grande longueur d'onde est très complexe et il est impossible de tenir compte de tous les détails de cette représentation dans les modèles numériques de circulation générale de l'atmosphère [1]. Il est donc nécessaire de développer des schémas approchés pour inclure dans ces modèles les échanges de chaleur dus au rayonnement. Une revue des différentes méthodes proposées pour calculer la divergence du flux radiatif de grande longueur d'onde a été faite par Stone et Manabé [2]. Paltridge et Platt [3] ont comparé les différents modèles de calculs de la divergence. Le principal critère de validité des résultats théoriques est évidemment la comparaison avec les résultats expérimentaux. Cox [4] a utilisé 180 sondages radiométriques réalisés pendant l'Année Internationale du Soleil Calme pour établir des modèles radiatifs liés aux caractéristiques synoptiques des latitudes moyennes. Fimpel, Kuhn et Stearns [5] ont comparé avec deux modèles les mesures faites pendant l'expérience GATE dans différents cas : situations de ciel clair, présence de cirrus, présence de nuages de basse et moyenne altitude.

Le Laboratoire de Météorọlogie Dynamique et le Laboratoire d'Energétique Solaire $\left({ }^{1}\right)$ ont proposé une approche statistique du problème de la paramétrisation des flux radiatifs de grande longueur d'onde. En comparant des mesures in situ de la divergence des flux radiatifs infrarouges aux caractéristiques des situations synoptiques, on espère établir une relation

(') Laboratoire d'Energétique Solaire, Odeillo, 66120 FontRomeu. paramétrique entre ces flux et les paramètres aérologiques classiques. On cherchera à mettre en évidence, plus particulièrement, l'influence de la vapeur d'eau et des nuages stratiformes de grande étendue.

Pour atteindre ce but, on se propose d'associer des mesures spectrométriques infrarouges et des mesures de flux radiatifs nets au sol à des sondages radiométriques verticaux comportant également la mesure précise des paramètres aérologiques classiques $(P, T, U)$. On réalisera une analyse détaillée de la situation synoptique à laquelle on adjoindra une analyse des images satellitaires (METEOSAT). Pour mettre au point la méthode, on commencera par des situations de ciel clair.

Les mesures radiométriques au sol seront réalisées à l'aide d'un spectroradiomètre à réseau analogue à celui déjà utilisé par C. Robert [6]. Ces mesures doivent permettre de déterminer le profil d'humidité par application d'une méthode d'inversion de l'équation de transfert radiatif. Pour cela, le programme de calcul de transmission de l'atmosphère raie par raie et couche par couche de N. Scott [7] sera utilisé.

La mise en évidence de l'influence de la vapeur d'eau sur les mesures radiométriques effectuées par sondage vertical nécessite un calcul théorique du flux de rayonnement susceptible d'être recueilli par le radiomètre visant soit vers le sol soit vers le haut de l'atmosphère.

La préparation des différentes mesures et des calculs qui leur sont associés est en cours et nous ne décrirons ici que la technique expérimentale utilisée pour obtenir les profils verticaux de température, d'humidité et de flux infrarouges entre le sol et le sommet de la troposphère. 
2. Dispositif expérimental. - Pour obtenir ces profils, on associe aux sondages météorologiques classiques sous ballons-sondes des mesures radiométriques dans l'infrarouge. Les mesures seront faites de nuit pour s'affranchir du rayonnement de courte longueur d'onde. Nous avons donc développé un radiomètre embarquable sous ballons; nous avons choisi et adapté à ces sondages une radiosonde utilisée dans le réseau météorologique international et nous avons mis au point une station de réception des données.

2.1 Mesures RADIOMÉTRIQUeS. - Les seuls radiomètres embarquables sous ballons-sondes et suffisamment économiques pour permettre des sondages de routine sont ceux qui ont été mis au point et utilisés par Suomi et al. [8]. Le radiomètre développé au laboratoire est basé sur le même principe en cherchant un compromis entre la sensibilité et le temps de réponse. Il est représenté schématiquement sur la figure 1 . Il est constitué de trois cellules identiques disposées à $120^{\circ}$ l'une de l'autre dans un disque de polystyrène expansé. Deux des cellules comportent un disque en mylar aluminisé peint en noir sur une de ses faces : l'une tournée vers le bas reçoit le rayonnement ascendant, l'autre, tournée vers le haut, le rayonnement descendant. La troisième cellule comporte un disque en mylar réfléchissant des deux côtés. La température de chacun des trois disques en mylar est mesurée à l'aide d'une thermistance subminiature collée sur la surface. Chaque face des disques de
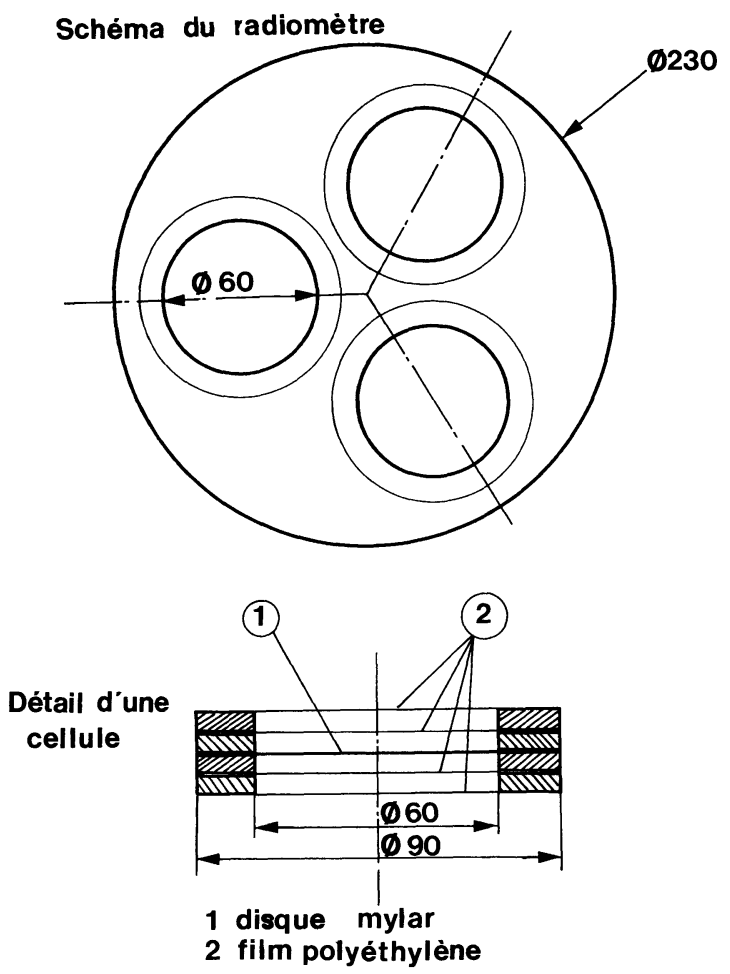

Fig. 1. - Schéma du radiomètre.

[Schematic diagram of the radiometer. $1:$ mylar disc ; 2 : polyethylene film.] mylar est protégée de la convection par deux feuilles de polyéthylène recuit de $25 \mu$ d'épaisseur.

La température de la cellule ayant le disque en mylar réfléchissant des deux côtés sert de température de référence; en effet, puisque les trois cellules sont identiques, cette température est celle de l'air dans les cellules en l'absence de rayonnement. Cette mesure est importante car elle permet de calculer les flux de façon simple et d'éviter d'écrire l'équation d'équilibre énergétique de l'appareil qui fait intervenir des termes de conduction et de convection difficile à définir avec précision.

Le temps de réponse du radiomètre est mesuré en laboratoire en plaçant l'appareil à $20 \mathrm{~cm}$ au-dessus d'un large cristallisoir rempli de glace fondante. $\mathrm{Ce}$ temps de réponse est de $30 \mathrm{~s}$.

Chaque appareil est étalonné au sol, face au ciel, par nuit claire, par comparaison avec un pyrradiomètre Eppley. On détermine ainsi pour chaque appareil un coefficient $\alpha$ défini par la formule :

$\alpha=\frac{T-T_{\mathrm{a}}}{F \downarrow-F \uparrow}$

dans laquelle : $T$ est la température du capteur radiométrique, $T_{\mathrm{a}}$ est la température de la cavité de référence, température de l'air; $F \downarrow$ le flux de rayonnement descendant; $F \uparrow$ le flux de rayonnement du corps noir à la température de l'air.

La sensibilité ainsi mesurée est peu variable d'un appareil à l'autre et de l'ordre de $7^{\circ} \mathrm{C}$ pour un flux de $100 \mathrm{~W} \mathrm{~m}^{-2}$.

Pour effectuer la mesure, lors d'un vol, les trois thermistances sont montées en diviseurs de tension, la tension étant ensuite transformée en fréquence par un V.C.O. Pour tenir compte des dérives en température, on ajoute une fréquence de référence qui correspond à une résistance infinie.

On a vérifié dans une enceinte à différentes températures que la fréquence à $+20^{\circ} \mathrm{C}$ et la fréquence à une température $T$ sont liées par la relation :

$\frac{f_{1,20^{\circ}}}{f_{1 T}}=\frac{f_{\mathrm{R} 20^{\circ}}}{f_{\mathrm{R} T}}$

où $f_{1}$ représente la fréquence correspondant à une thermistance de mesure et $f_{\mathbf{R}}$ la fréquence de référence. Pour la mesure, on se ramène à la fréquence d'étalonnage à la température ambiante en appliquant cette relation. A cause de la rapidité des dérives, on a été amené à fixer la séquence suivante de mesure : référence, thermistance 1 , thermistance 2 , référence, thermistance 3 . On transmet donc 5 fréquences pour mesurer 3 températures.

2.2 LES RADIOSONDES. - Le choix de la radiosonde a été déterminé d'une part par la qualité des capteurs, leur sensibilité et leur temps de réponse, d'autre part par la possibilité de télémesurer, d'une façon simple, des capteurs supplémentaires, c'est-à-dire les données 
des radiomètres. Une comparaison des sondes française, américaine et finlandaise nous a amené à choisir cette dernière. Le contacteur à entraînement mécanique qui assure la transmission successive des différents paramètres peut être remplacé par un dispositif électronique permettant l'introduction de paramètres supplémentaires et le choix de la cadence d'échantillonnage.

Le baromètre et le thermomètre de la radiosonde Vaisala sont associés à des condensateurs plans dont la capacité varie avec la distance des armatures. Pour le baromètre, c'est la déformation d'une capsule anéroïde qui fait varier cette distance et pour le thermomètre, c'est la déformation d'un bilame. L'hygromètre est constitué par un film mince de polymère organique qui adsorbe l'eau. Ce film constitue le diélectrique d'un condensateur dont la capacité varie avec la quantité d'eau adsorbée. Ainsi, chacun des capteurs de la radiosonde contrôle la capacité d'un condensateur, cette capacité détermine à son tour la fréquence du signal transmis. La fréquence transmise est donc fonction de $P, T, U$. En plus des trois capteurs atmosphériques, la radiosonde comporte deux condensateurs de capacité constante qui fournissent deux fréquences de référence $f_{1}$ et $f_{2}$ permettant de tenir compte des différentes dérives.

La fréquence $f$ du signal de la radiosonde est exprimée par la formule générale :

$f=\frac{1}{2 \pi \sqrt{L C}}$

dans laquelle $L$ est l'inductance de l'oscillateur, $C$ la capacité.

On définit la quantité $Y$ par la relation :

$Y=\frac{\left(1 / f_{2}\right)^{2}-\left(1 / f_{\mathrm{e}}\right)^{2}}{\left(1 / f_{2}\right)^{2}-\left(1 / f_{1}\right)^{2}}$

où $f_{\mathrm{e}}$ représente la fréquence correspondant au capteur, soit : $f_{\mathrm{p}}, f_{\mathrm{v}}, f_{\mathrm{u}} ; f_{1}$ et $f_{2}$ sont les fréquences de référence.

Les différents capteurs ont été étalonnés au laboratoire à travers toute la chaîne de mesure en fonction du paramètre $Y$. Les courbes représentées sur la figure 2 donnent des exemples de ces étalonnages pour une radiosonde.

Une mesure faite au hertz près entraîne la précision suivante pour les trois capteurs :

$$
\begin{aligned}
& \text { Pression : } \quad 6 \mathrm{mb} \text { à } 1000 \mathrm{mb} \\
& 2,6 \mathrm{mb} \text { à } 600 \mathrm{mb} \\
& 0,6 \mathrm{mb} \text { à } 200 \mathrm{mb} \text {. }
\end{aligned}
$$

Température : $0,4^{\circ} \mathrm{C}$

Humidité : $1 \%$.

2.3 TRANSMISSION ET RÉCEPTION DES DONNÉES. - La commutation des capteurs, représentée sur la figure 3, se fait par l'intermédiaire de relais reliés successivement à un oscillateur pour les capteurs de la radio-

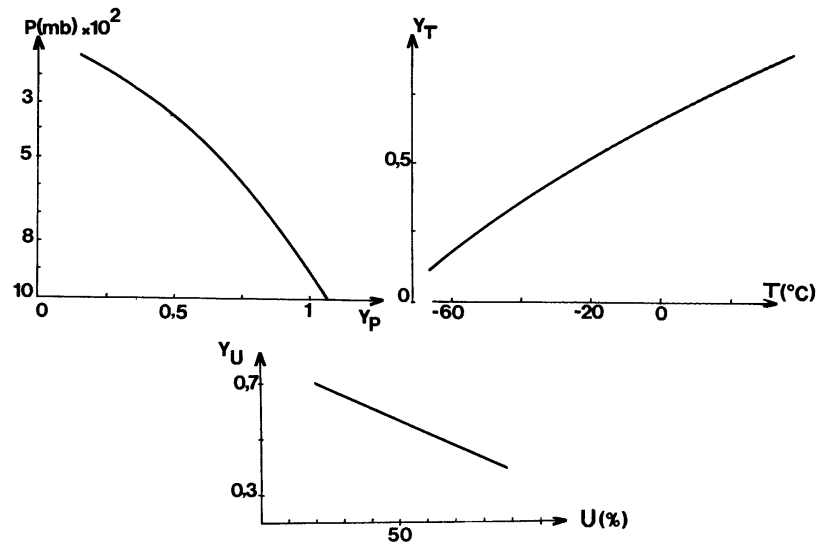

Fig. 2. - Courbes d'étalonnage de la radiosonde.

[Calibration curves of the radiosonde.]

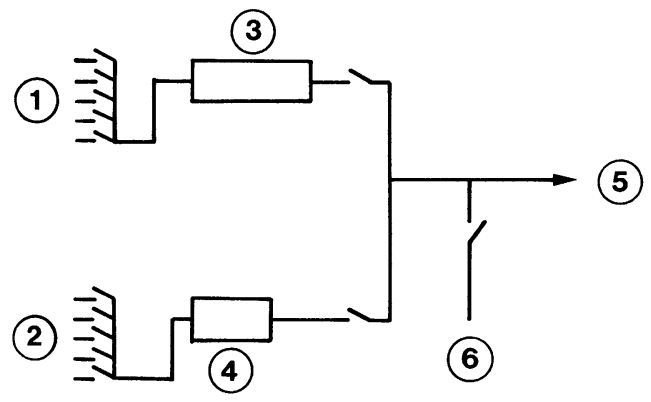

Fig. 3. - Représentation schématique de la commutation des paramètres : 1 : relais capteurs radiosonde ; 2 : relais capteurs radiomètre; 3 : oscillateur ; 4 : V.C.O.; 5 : émetteur; $6:$ relais commande séquence.

[Schematic diagram of the switching of the parameters $: 1:$ radiosonde relays; 2 : radiometer relays; 3 : oscillator; 4 : V.C.O.; $5:$ transmitter ; 6 : relay command sequence.]

sonde et à un V.C.O. pour les capteurs du radiomètre. L'horloge de commande permet d'obtenir d'une part un silence entre les différents paramètres, et d'autre part un silence plus long pour marquer le début de la séquence de passage des paramètres. Elle offre le choix de quatre périodes possibles : $0,3 \mathrm{~s}, 0,6 \mathrm{~s}$, $1,2 \mathrm{~s}$ et $2,4 \mathrm{~s}$ pour le passage de chaque paramètre. On transmet au total onze paramètres : 5 pour la radiosonde, 5 pour le radiomètre et un silence. En choisissant à priori la période de $0,6 \mathrm{~s}$, le temps de passage de ces onze paramètres est inférieur à $7 \mathrm{~s}$, soit, en supposant une vitesse ascensionnelle du ballon de $5 \mathrm{~m} / \mathrm{s}$, une mesure complète tous les $35 \mathrm{~m}$.

La réception se fait avec un récepteur $400 \mathrm{MHz}$ à modulation de fréquence qui recrée le créneau de départ. Le comptage se fait pendant $0,1 \mathrm{~s}$ et on compte la fréquence multipliée par 10. Le récepteur est donc associé à un amplificateur et à un multiplicateur par 10 qui entraîne le déclenchement d'une imprimante.

3. Essais technologiques. - Pour contrôler le fonctionnement de la sonde radiométrique, deux 
essais technologiques ont été faits au mois de mars 1978 à Odeillo, la nuit, par situation de ciel clair.

Le radiomètre est placé à $60 \mathrm{~m}$ en dessous du ballon pour minimiser le rayonnement de celui-ci sur les capteurs. La radiosonde est pendue à $20 \mathrm{~m}$ sous le radiomètre. Avant le lancer, les valeurs des paramètres aérologiques sont contrôlés par comparaison avec des appareils au sol : un baromètre Fortin pour la pression, une sonde au platine convenablement ventilée pour la température et un psychromètre pour l'humidité.

Les conditions météorologiques des deux vols de mars 1978 étaient tout à fait analogues. Les figures 4 et 5 montrent, pour un des vols, respectivement le sondage aérologique et les profils verticaux de flux infrarouges. On a obtenu des mesures toutes les $7 \mathrm{~s}$ environ, ce qui donne un sondage presque continu puisque la vitesse ascensionnelle du ballon était en moyenne de $5 \mathrm{~m} / \mathrm{s}$.

Les valeurs du flux net $R_{\mathrm{n}}$, comparées à celles de Kuhn et Johnson [9] ou à celles obtenues au cours de l'expérience Gate [5], semblent trop élevées dans les couches supérieures de la troposphère. Ce résultat pourrait être expliqué par une non-linéarité de la réponse du radiomètre. En effet, un étalonnage par

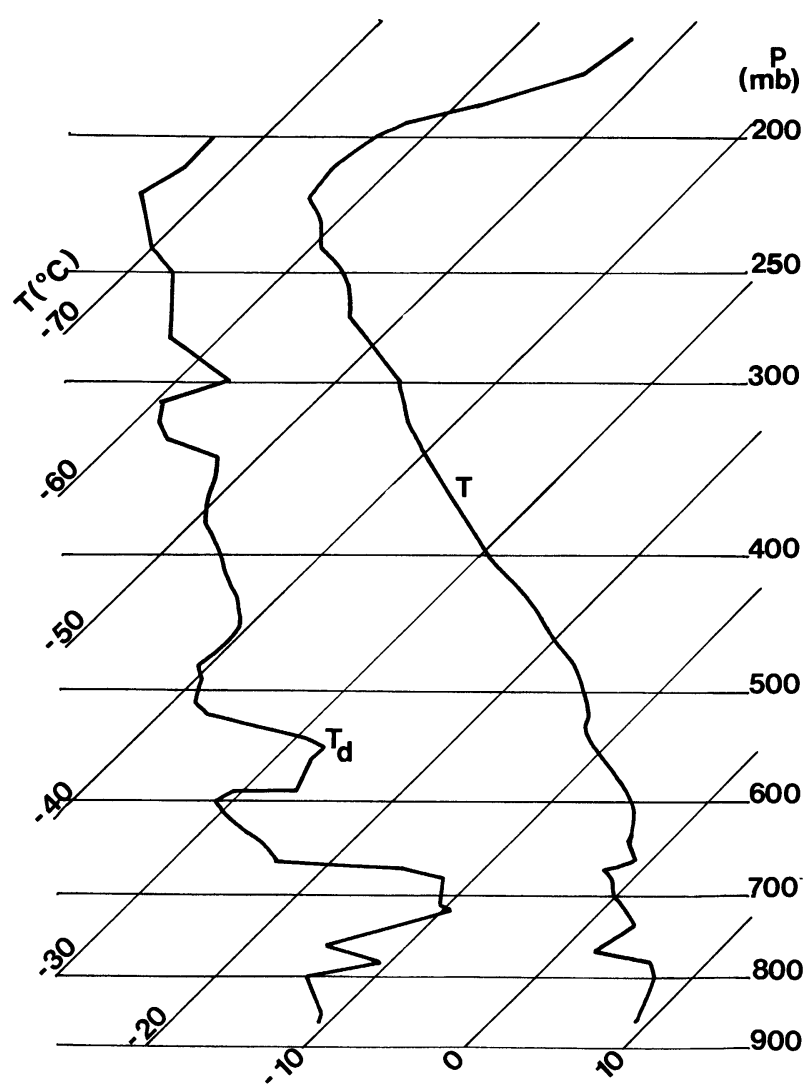

Fig. 4. - Sondage du 10 mars 1978. Odeillo, 21 h T.U.

[Sounding, March 10, 1978, Odeillo, 9 h.p.m. G.M.T.]

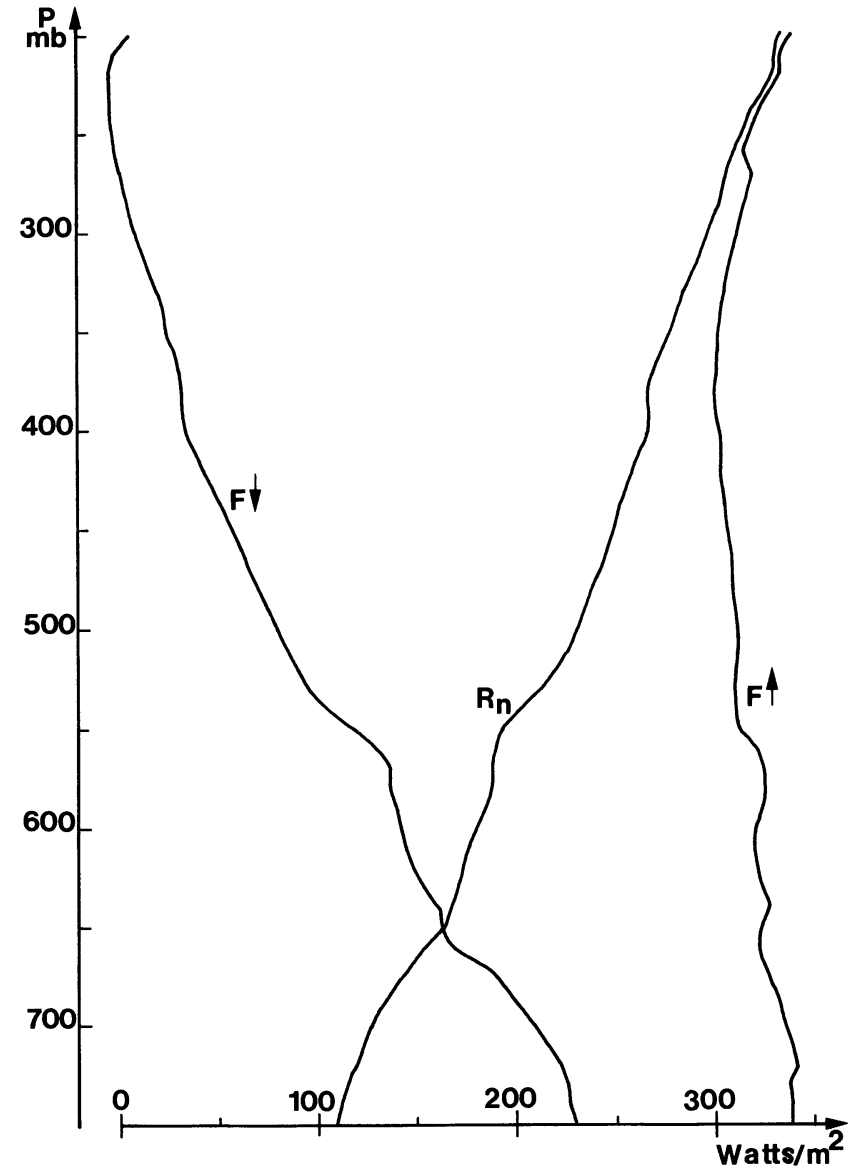

Fig. 5. - Profils verticaux des flux infrarouges le 10 mars 1978 , Odeillo, 21 h T.U.

[Infrared flux profiles, March 10, 1978, Odeillo, 9 h.p.m. G.M.T.]

comparaison avec un appareil au sol ne permet de définir le coefficient $\alpha$ de l'appareil qu'à la température ambiante. Nous nous proposons donc d'améliorer l'étalonnage des radiomètres en les plaçant devant un corps noir dont on pourra faire varier la température de rayonnement.

4. Conclusion. - Après avoir exposé le projet concernant l'étude de l'influence de la vapeur d'eau sur les flux radiatifs de grande longueur d'onde, on a décrit en détail le dispositif utilisé pour les sondages radiométriques de la trophosphère.

Dans le courant de l'année à venir, deux campagnes de mesures, groupant les différents dispositifs expérimentaux, vont être faites sur des sites permettant d'avoir une surface de base de température et d'émissivité aussi homogène que possible. On cherchera des situations météorologiques permettant d'effectuer des mesures dans une gamme suffisamment étendue de profils de température et d'humidité. 


\section{Bibliographie}

[1] Kondratiev, K. Ya., W.M.O. no 309 (1972).

[2] Stone, H. M., Manabe, S., Month. Weather Rev. 96 (1968) 735-742.

[3] Paltridge, G. W., Platt, C. M. D., Radiative Processes in Meteorology and Climatology (Els. Publ. Co.) 1976, 179.

[4] Cox, S. K., Month. Weather Rev. 97 (1969) 637-651.

[5] Fimpel, H. P., Kunn, P. M., Stearns, L. P., Symposium on Radiation in the Atmosphere (1977) 533-535.
[6] Robert, C., Thèsé de $3^{\text {e }}$ Cycle, Paris (1976).

[7] Scotr, N. A., J. Quant. Spectrosc. Rad. Transf. 14 (1974) 691.

[8] Suomi, V. E., Staley, D. O., Kuhn, P. M., Quart. J. Roy. Met. Soc. 84 (1958) 134-141.

[9] Kuhn, P. M., Johnson, D. R., J. Geophys. Res. 71 (1966) $367-$ 373. 